Hans $\mathrm{K} l$ in $\mathrm{k}$

\title{
Die Bankgehilfenprüfung in programmierter Form
}

\section{Auflage}

In die Abschlußprüfungen zum Bankkaufmann vor den Industrie- und Handelskammern finden die programmierten Fragen immer mehr Eingang. $\mathrm{Zu}$ jeder der Fragen sind dann meist mehrere Antworten vorgegeben, von denen der Prüfling die richtige(n) herausfinden und ankreuzen muß. Die Lösungen werden auf einen Lösungsbeleg übertragen, der durch einen Computer ausgewertet wird.

Von den Prüflingen wird dieses moderne Prüfungsverfahren durchweg begrüßt, so daß die erste Auflage dieses Buches als große Hilfe angesehen wurde. Innerhalb von sechs Jahren wurden deshalb auch vier Auflagen an sich auf die Prüfungen vorbereitende Bankauszubildende verkauft.

Der Verfasser, der zusammen mit seinem Co-Autor Erich Hüttner auch programmierte Fragen zum Bankrechnungswesen und zum Bankrechnen verfaßt hat, legt mit diesem Buch die fünfte überarbeitete Ausgabe seiner programmierten Fragen zur Bankgehilfenprüfung vor und kann damit sicher sein, daß dieses Werk weiterhin helfen wird, den Prüfungskandidaten nach gründlicher Durcharbeitung die Angst vor den Lehrabschlußprüfungen zu nehmen. Selbstverständlich ist dieses Buch auch als Repetitorium für diejenigen Prüflinge geeignet, welche die Prüfung nicht nach dem Normtestverfahren ablegen.

Betriebswirtschaftlicher Verlag Dr. Th. Gabler, Wiesbaden 


\section{Klink}

Die Bankgehilfenprüfung

in programmierter Form 
Zur Vorbereitung auf die Fächer "Bankbuchführung" und "Bankrechnen" empfehlen wir die ebenfalls in unserem Verlag erschienenen Bücher:

Erich Hüttner / Hans Klink:

Bankrechnungswesen in programmierter Form

ISBN 3409430245

Erich Hüttner / Hans Klink:

Bankrechnen in programmierter Form

ISBN 3409470441 


\title{
Die Bankgehilfenprüfung in programmierter Form
}

\author{
Wiederholungs- und Übungsbuch \\ von
}

Hans Klink

Fünfte, durchgesehene Auflage

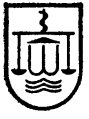

Springer Fachmedien Wiesbaden $\mathrm{GmbH}$ 
ISBN 978-3-409-81821-6 ISBN 978-3-663-1 4804-3 (eBook)

DOI 10.1007/978-3-663-14804-3

Copyright by Springer Fachmedien Wiesbaden 1977

Ursprünglich erschienen bei Betriebswirtschaftlicher Verlag Dr. Th. Gabler, Wiesbaden 1977 


\section{Vorwort zur ersten Auflage}

Vor wenigen Jahren wurde in der Bundesrepublik Deutschland der Versuch unternommen, Abschlußprüfungen in programmierter Form durchzuführen. Der Versuch gelang; heute führen bereits mehrere Industrie- und Handelskammern die Bankgehilfenprüfung nach dem Normtest-Electronic-System durch. Die Zeit wird kommen, wo alle Industrie- und Handelskammern die gesamten Abschlußprüfungen nur noch in programmierter Form durchführen werden.

In der programmierten Abschlußprüfung werden zu jeder Frage mehrere Antworten vorgegeben. Der Prüfling hat nun die Aufgabe, die richtige Antwort herauszufinden und diese anzukreuzen. Die Lösungen sind dann auf einen Lösungsbeleg zu übertragen, der durch einen Computer ausgewertet wird.

Von den Prüflingen wird dieses moderne Prüfungsverfahren durchweg begrüßt, wenngleich andererseits von ihnen bemängelt wird, daß entsprechendes Obungsmaterial nur spärlich zur Verfügung steht.

Mit diesem Abriß wird dem Mangel an geeignetem Obungsmaterial abgeholfen.

Zu jeder der 511 Fragen werden drei Anworten vorgegeben; zwei davon sind falsch. Manchmal hängt die richtige Antwort nur von einem einzigen Wort oder einer einzigen Zahl ab. Welche Antwort die richtige ist, wird jeweils auf der folgenden Seite angegeben. Der Prüfungskandidat sollte zunächst selbst den Versuch unternehmen, die zutreffende Antwort zu finden. Erst danach sollte festgestellt werden, welche von den drei Alternativantworten die richtige ist.

Im übrigen darf wohl gesagt werden, daß sich dieses Buch auch als Repetitorium für diejenigen Prüflinge eignet, welche die Prüfung nicht nach dem Normtest-Verfahren ablegen. 


\section{Vorwort zur fünften Auflage}

Innerhalb von sechs Jahren nach Erscheinen der ersten Auflage kann die fünfte Auflage vorgelegt werden.

Das ist - so meine ich - u. a. ein Beweis dafür, daß ein echtes Bedürfnis für die Darbietung des Prüfungsstoffes in dieser Form vorliegt.

Möge das Buch auch weiterhin eine gute Verbreitung finden und somit dazu beitragen, den Prüfungskandidaten nach gründlicher Durcharbeitung die Angst vor der Lehrabschlußprüfung zu nehmen.

Der Verfasser 


\section{Inhaltsverzeichnis}

1. Geld - Währung - Notenbankwesen 9

2. Die Buchführung der Kreditinstitute 29

3. Das Konto als Grundlage der Geschäftsverbindung 51

$\begin{array}{ll}\text { 4. Der Sparverkehr } & 73\end{array}$

5. Das prämienbegünstigte Sparen und das Sparen nach dem Vermögensbildungsgesetz $\quad 91$

$\begin{array}{ll}\text { 6. Der Scheckverkehr } & 105\end{array}$

$\begin{array}{ll}\text { 7. Der Wechselverkehr } & 129\end{array}$

8. Der Oberweisungs- und Lastschriftverkehr 159

9. Die Wertpapiere 175

10. Das Depotgeschäft 195

11. Der Wertpapierhandel 219

12. Das Emissionsgeschäft 241

13. Der Sortenhandel 253

14. Die Devisen- und Außenhandelsgeschäfte 267

15. Das Kreditgeschäft 293

$\begin{array}{ll}\text { Sachwortverzeichnis } & 321\end{array}$ 